\title{
Developing Dually Optimal LCA Features in Sensory and Action Spaces for Classification
}

\author{
Nikita Wagle and Juyang Weng \\ Department of Computer Science \& Engineering \\ Michigan State University \\ East Lansing, Michigan 48824 \\ Email: \{waglenik,weng\}@msu.edu
}

\begin{abstract}
Appearance based methods have utilized a variety of techniques such as Linear Discriminant Analysis (LDA), Support Vector Machine (SVM), k-means clustering, and sparse autoencoders to extract training-data dependent features from static images - by static images, we mean, foreground objects and backgrounds are present in static images as snapshots. The Developmental Networks (DN) use Lobe Component Analysis (LCA) features developed not only from the image space $X$ but also the action space $Z$. Since, $Z$ can be taught to represent a set of trainer specified meanings (e.g., type and location), a DN treats these meanings in a unified way for both detection and recognition for objects in dynamic cluttered backgrounds. However, the DN method has not been applied to publicly available datasets and compared with well-known major techniques. In this work, we fill this void. We describe how the $Z$ information enables the features to be more sensitive to trainer specified output meanings (e.g., type and location). The reported experiments fall into two extensively studied categories - global template based object recognition and local template based scene classification. For the datasets used, the performance of the DN method is better or comparable to some major local template based methods but the DNs also provide statistics-based location information about the object in a cluttered scene.
\end{abstract}

\section{INTRODUCTION}

Techniques for appearance-based pattern recognition can be categorized into two types - global template based methods and local template based methods. The former type assumes that the object of interest has already been detected and cropped, then shifted, scaled, and rotated in a standard way. The later type uses multiple local templates at arbitrary image locations on scenes where features of objects of interest can arise anywhere in the scene. If the location of object is not reported, the later type is also called scene classification (i.e., features indicate a scene type).

\section{A. Existing Major Methods}

One of the major contributions of this work is the sources from which features are extracted (or developed). The traditional methods discussed here, such as LDA, SVM, space auto-encoders, sparse RBM, k-means clustering, etc. typically extract features from sensory (i.e. image) space only, not including the action (i.e. motor) space, and the feature space (i.e. feature response competition) itself. The sparse method such as k-means clustering, finds top-k clusters; however, learning is not based on top-k mechanism, but on the nearest cluster instead in a batch fashion. In this work, the network
TABLE I

COMPARISON OF CHARACTERISTICS OF GLOBAL AND LOCAL TEMPLATE BASED METHODS. SPARSE METHODS INCLUDE SPARSE AUTO-ENCODERS, SPARSE RBMS, K-MEANS CLUSTERING, ETC. IN THIS WORK.

\begin{tabular}{lcccc}
\hline Characteristics & LDA & SVM & Sparse Methods & DN \\
\hline \hline Environmental openness & Possible & No & No & Yes \\
High dimensional sensors & Yes & Possible & Yes & Yes \\
Completeness & Yes & No & No & Yes \\
Real-valued actions & No & No & No & Yes \\
Real-time training & No & No & No & Yes \\
Incremental learning & No & Yes & No & Yes \\
Perform while learning & No & No & No & Yes \\
Input having background & No & No & Yes & Yes \\
\hline
\end{tabular}

structure of the DN is at least as important as the feature clustering techniques.

The LDA technique [1], [2] finds a linear feature space in which the ratio of the between-class scatter over the with-class scatter is maximized. The SVM technique [3], [4] constructs a hyperplane in a high-dimensional kernel feature space, so that the margin to the nearest data points is maximized. The local template based methods use features derived from local patches of images (rather than from the whole image) by applying well-known unsupervised feature learning algorithm such as sparse auto-encoders [5], sparse RBM [6], k-means clustering, etc. [7], [8], [9].

\section{B. Characteristics of $D N$}

The DN (Developmental Network) framework [10] has used global templates from image datasets [10], as well as local templates from cluttered scenes [11].

The novel characteristics of a DN over some well-known pattern recognition methods are discussed below and a summary is provided in Table I.

1) Environmental openness: The DN learns and improves from human taught with motor-supervised experience of actions through realistic scenes, though its performance is imperfect due to limitations in computational resource and learning experience. The DN does not assume that only a few objects (e.g., face) are of interest, so the environment is open.

2) Low and high dimensional sensors: The DN is applicable to low- and high-resolution video, but should work reasonably well regardless of the resolution, since the internal operations of DN are based on normalized inner product.

3) Completeness: All the features in DN emerge as optimal 
representations learned from the different amounts of teaching. The DN does not use, and not limited by, handcrafted features (e.g., SIFT, or oriented edges which may fail if edges are sparse or absent in an input).

4) Traditionally, only class labels are used as output, but DN framework enables the system to learn multiple concepts so that "type" is just one among other possible concepts such as "location" and "scale" (not experimented here).

5) The DN framework uses top-down input in the $Z$-area representing "location" and "type" information, as well as the traditional bottom-up inputs in the $X$-area to develop optimal features. Such new kind of features is able to disregard input dimensions that are irrelevant to top-down inputs [10].

6) The DN finds optimal clusters in the space of $X \times Z$, (instead of only image space $X$ ), which are sensorimotor features, where $Z$ is the effector space. By optimal, we mean maximal likelihood (ML) in the representation of the space of $X \times Z$ is based on limitation in computational resource amount of teaching in DN.

7) Real-valued actions: The DN accepts and processes real-valued sensory and motor information, instead of human supplied discrete class labels (e.g., each neuron in $Z$ represents a class), making it more general, since actions can be taught or created for the physical world without a human predefined class.

8) Real-time training: During training, the sensory and memory refreshing rate must be high enough so that each physical event can be temporally sampled and processed in real-time. The class labels of objects appearing in the real scene require a human to supply, restricting the possibility of real-time training. The DN can take actions directly, regardless of whether they represent a real-valued action or a class label.

9) Incremental learning: The DN supports batch learning. Additionally, acquired skills must be used to assist in the acquisition of new skills, as a form of "scaffolding." Each new observation $\mathbf{x} \in X$ and $\mathbf{z} \in Z$ must be used to update the current DN and the current $(\mathbf{x}, \mathbf{z})$ must be discarded before the next $(\mathbf{x}, \mathbf{z})$ can be acquired. The amount of data in the sensory and motor stream is virtually unbounded in real time, making incremental learning necessary.

10) Perform while learning: At any time, the human teacher can teach the DN by imposing an action on its motor port $Z$ (motor-supervised learning). As soon as the human lets $Z$ free, the DN generates $\mathbf{z} \in Z$ as its best prediction or best action at this time.

11) Input having background: The DN can deal with object of interest in any position in unknown cluttered backgrounds. When $\mathrm{DN}$ is trained on local patches derived from image scenes, they add "votes" to a single supervised location in the Location Motor (LM) of $Z$, although different local patches are at different locations in the input image.

\section{Novelty}

This work has two major novelties - First, the DN algorithm is originally meant for a more general problem of spatiotemporal event detection and recognition in complex background, and is not restricted to the global and local template based image matching problem. However, it is desirable that we apply it to the space-only problems here so that it can be compared with major pattern recognition techniques. The ways in which motor space $Z$ is used, are different between DN method and others compared. Second, this work is the first to use the DN for problems where multiple local features contribute to the classification (type) and localization (location). Multiple firing feature detectors ( $Y$ neurons) vote for the corresponding single neuron in the Type Motor (TM) area and the corresponding single neuron in the Location Motor (LM) area, the two subareas of area $Z$.

DN can be used for shallow learning, deep learning, and a mixed shallow and deep learning. Here, we concentrate on shallow learning. Experimentally, we show that the shallow learning (DN) is at least comparable to the deep learning methods (sparse auto-encoders, k-means clustering, etc.). In a local feature based DN, the "where" information from LM is applied as supervised input to the DN along with the "what" information during the training phase. While $Z$ is free during testing phase, through multiple updates of DN, the location and type information fed from $Y$ to $Z$ and then from $Z$ back to $Y$ reinforce each other and suppress inconsistent features - the object location must be consistent with type and vice versa. Thus, shallow learning method DN has faster relaxation between $Y$ and $Z$ than in deep learning methods.

The remainder of the paper is organized as follows. In section II, we review previous work. In section III, we explain DN. The experimental results are presented in section IV, and section $\mathrm{V}$ gives concluding remarks.

\section{Previous Work}

In this section, we briefly discuss the most widely applied algorithms for global and local template based image matching.

\section{A. Global Template Based Methods}

Of the many methods for global template matching (Wavelets, Neural Networks, Correlation, PCA, LDA, SVM), we discuss two types of methods - LDA and SVM.

1) LDA algorithm: The LDA algorithm, previously devised in [2] is based on optimal subspace generation using two projections - a Karhunen Loéve projection to produce a set of MEF (most expressive features) features followed by a discriminant analysis projection to produce a set of MDF (most discriminant features) features. The MEF projection is sensitive to lighting variations. The MDF overcomes this limitation by using information in the class labels.

2) SVM algorithm: The SVM algorithm [3], [4], maps a set of n-dimensional data points from a finite-dimensional space to a high-dimensional space, constructing a (n-1)-dimensional maximum- margin hyperplane or set of hyperplanes in a highdimensional space, so that a functional margin, that has the largest distance to the nearest training data points of any class, is maximized. The testing data points are then mapped to the same space and are predicted to belong to a class based on 


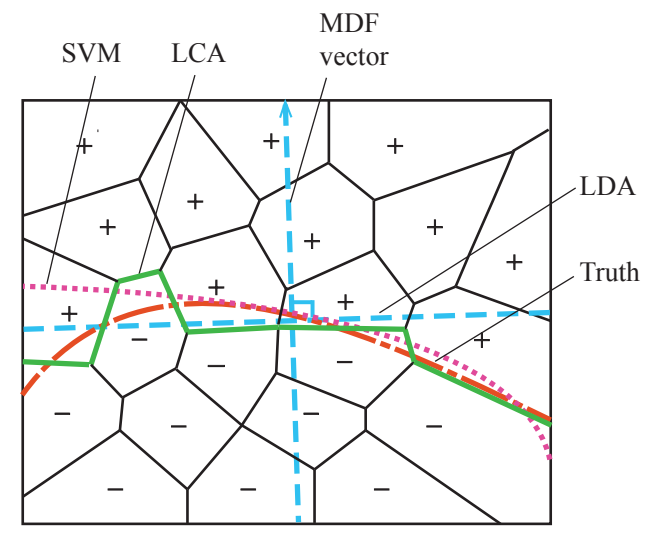

Fig. 1. The two-class clustering of data points when subject to linear separator in MDF space in LDA algorithm, RBF kernel based non-linear SVM classification versus the separation of data points into voronoi regions in DN algorithm

which side of the margin they fall on. When relation between data points and class labels is non-linear, the Gaussian RBF kernel $\left(K\left(x_{i}, x_{j}\right)=\exp \left(-\gamma\left\|x_{i}-x_{j}\right\|^{2}\right), \gamma>0\right)$ is chosen over the linear kernel. The effectiveness of an RBF kernel depend on a good choice of two parameters $\mathbf{C}$ and $\gamma$, where $\mathrm{C}>0$ is the penalty parameter of degree of misclassification and $\gamma=\frac{1}{2 \sigma}$, and $\sigma$ determines the area of influence of the support vector over the new data instance.

Figure 1 shows the two-class clustering of data points the LDA boundary is linear, whereas the SVM boundary is characterized by the non-linear kernel function chosen. The DN algorithm separates data points into voronoi regions, such that the boundary separating the two regions is at an equal distance from both points.

\section{B. Local Template Based Methods}

A simple feature learning framework that incorporates a local feature-learning algorithm like sparse auto-encoders [5], sparse RBMs [6], K-means clustering, and Gaussian mixture models [7], [8], as a "black box" module [9] within has been studied to discover local features from unlabeled data instances. A set of random patches is extracted from unlabeled training data instances, such that each patch has dimension $w \times w$ and has d-channels, where $w$ is the size of the receptive field; each $w \times w$ patch can be represented as a vector in $\mathbb{R}^{N}$ of pixel intensity values, such that $N=w \cdot w \cdot d$. A dataset of randomly sampled patches $X=x^{(1)}, \ldots, x^{m}$ is then constructed, where $x^{i} \in \mathbb{R}^{N}$. Then, each patch $x^{(i)}$ is normalized, and the dataset is optionally whitened. An unsupervised learning algorithm viewed as a "black box" module takes the dataset $X$ and outputs a function $f: \mathbb{R}^{N} \rightarrow \mathbb{R}^{K}$ and maps input vector $x^{(i)}$ to a new feature vector $y=f(x) \in \mathbb{R}^{K}$ of $K$ features, where $K$ is a parameter of the unsupervised learning algorithm used. A $(n-w+1) \times(n-w+1)$ representation with $K$ channels is defined for each $w \times w$ "subpatch" of the training data; $y^{(i, j)}$ is referred to as the $K$-dimensional feature representation extracted from location $i, j$ of the training data.
The feature representation, $y^{(i, j)}$ is split into four equally sized quadrants and the sum in each quadrant is computed to yield a reduced $K$-dimensional representation of each quadrant for a total of $4 K$ features on which a linear classification algorithm is applied to identify new data instances [9].

\section{The Developmental Network Model}

In this work, we consider the DN model to have a general purpose brain area $Y$, which is connected with the sensory area $X$ and the motor area $Z$, as illustrated in Figure 2, in which the order of areas from low to high is $X, Y, Z$. Much of the material in this section in extracted from Weng and Luciw 2011 [10].

\section{A. Area Function}

At the first $c$ time instances, during the "prenatal" learning phase, the first $c$ neurons of $A$ in $\{X, Y, Z\}$ initialize their synaptic vectors $V=\left(\mathbf{v}_{1}, \mathbf{v}_{2}, \ldots, \mathbf{v}_{c}\right)$, where each synaptic vector $\mathbf{v}_{i}$ is initialized using the input pair $\mathbf{p}_{i}=\left(\mathbf{b}_{i}, \mathbf{t}_{i}\right)$, where the bottom up input is $\mathbf{b}_{i}$ and the top-down input is $\mathbf{t}_{i}$, $i=1,2, \ldots, c$, and initialize the firing ages $A=\left(a_{1}, a_{2}, \ldots, a_{c}\right)$, such that each firing age $a_{i}$ is initialized to be zero, $i=$ $1,2, \ldots, c$. After the "birth" phase, at each time instant, each area $A$ computes its response $\mathbf{r}^{\prime}$ from its input pair $\mathbf{p}=(\mathbf{b}, \mathbf{t})$ based on its adaptive part $N=(V, A)$ and its current response $\mathbf{r}$. The current response $\mathbf{r}$ is regulated by the attention vector $\mathbf{b}_{a}$. Each area $A$ updates its adaptive part $N$ to $N^{\prime}$ as follows:

$$
\left(\mathbf{r}^{\prime}, N^{\prime}\right)=f\left(\mathbf{b}, \mathbf{r}, \mathbf{t}, \mathbf{r}_{a}, N\right)
$$

where $f$ is the area function. The attention supervision vector $\mathbf{r}_{a}$ is used to softly avoid the area $A$ from excessively learning background; it suppresses all the $A$ neurons to zeros except $3 \times 3=9$ ones centered at the correct object location. The area $A$, whether $X, Y$, or $Z$ compute and update in a unified way as described above. But $X$ area does not have bottom-up input and $Z$ area does not have top-down input, since they are nerve terminals.

\section{B. Area Computation}

We introduce that the receptive field (RF) of a neuron should contain three parts - sensory RF (SRF), motor RF (MRF) and lateral RF (LRF), respectively. The effective field (EF) of each neuron should also include three parts: sensory EF (SEF), motor EF (MEF), and lateral EF (LEF), respectively. The SRF, MRF, LRF, SEF, MEF, LEF together form the hextuple fields of a neuron as shown in Figure 4(a). The lateral connections - connections with neurons in the same area that are strongly correlated or anticorrelated, the highly correlated cells are connected by excitatory connections to generate a smooth map that globally covers a rough "terrain" but gradually becomes selective and local to fit the details of the "terrain", whereas, highly anti-correlated cells are connected by inhibitory connections, so that the neurons in the same cortical area can respond to different features, and allow only top ranked neurons to fire and update, ensuring that other weakly responding neurons do not fire so that they do not learn 
irrelevant information and also keep their long-term memory intact.

Consider an area $A$ in $\{X, Y, Z\}$. Each neuron in $A$ has a weight vector $\mathbf{v}=\left(\mathbf{v}_{b}, \mathbf{v}_{t}\right)$, corresponding to the two area inputs $(\mathbf{b}, \mathbf{t})$ if both inputs are associated with them, else the part of input that is not associated with the area is not included in the notation. The sum of two normalized inner products gives the pre-action of the neuron:

$$
r\left(\mathbf{v}_{b}, \mathbf{b}, \mathbf{v}_{t}, \mathbf{t}\right)=\dot{\mathbf{v}} \cdot \dot{\mathbf{p}},
$$

where $\dot{\mathbf{v}}$ is the unit vector of the normalized synaptic vector, $\mathbf{v}=\left(\dot{\mathbf{v}}_{b}, \dot{\mathbf{v}}_{t}\right)$, and $\dot{\mathbf{p}}$ is a unit vector of the normalized input vector, $\mathbf{p}=(\dot{\mathbf{b}}, \dot{\mathbf{t}})$. The inner product measures the degree of match between the two directions $-\dot{\mathbf{v}}$ and $\dot{\mathbf{p}}$, because $r\left(\mathbf{v}_{b}, \mathbf{b}, \mathbf{v}_{t}, \mathbf{t}\right)=\cos (\theta)$ where $\theta$ is the angle between two unit vectors $\dot{\mathbf{v}}$ and $\dot{\mathbf{p}}$. In our DN based LCA algorithm [10], we use top-k mechanism to show that lateral connections within neurons in each area enable them to sort top winner neurons within each time step $t_{n}, n=1,2,3, \ldots$ If $k=1$, only one winner neuron fires with a response value $y_{j}=1$ and the rest of the neurons in the area do not fire, so $y_{i}=0$ for $i \neq j$. Thus, in general, for $k>1$, a dynamic scaling function shifts and scales the pre-action potential of each neuron $r_{i}$ in a dynamic manner, so that the winner has a response value $y=1$ and the $(k+1)$-th and weaker neurons have a response value zero.

\section{Dual Optimality of Lobe Component Analysis}

The Lobe Component Analysis (LCA) [10] is a model for long-term memory retention, and uses a dually optimal (optimal in space and time) framework that casts long-term and short-term memory together by the optimal distribution of the limited number of neurons of each area in the input space $X \times Z-$ optimal Hebbian learning, as shown in Figure 3 .

In LCA framework, the $Y$ area acts as a bridge between the sensory area $X$ and the motor area $Z$, as shown in 2. The twoway local connections in green represent neuronal input and in pink represent neuronal output. In the same area, near neurons are connected by excitatory connections for smoothness of representation, and far neurons are connected by inhibitory connections, hence, competition between neurons result in detection of different features by different neurons. The meaning of the dual (spatiotemporal) optimality of LCA is shown in Figure 3. The upper area is a 2-D representation of the positions for the neurons in several stacked layers within $Y$ area. The top-3 firing neurons in the $Y$ area - top-1 neuron in dark blue shade at the center, top-2 in light blue shade, and top- 3 in vertical blue $\mathrm{n}$ white stripes, are contextdependent working memory for the current context and the ones that do not fire are context dependent long-term memory for the current context. A 2-D representation of a very high dimensional input space $P=X \times Z$ of the cortical area $Y$ is shown by the lower area, in which each neuron in $Y$ plane is linked with its synaptic weight vector by a curved arc; the synaptic weight vectors of $Y$ represented in $P$ as small dots define a Voronoi diagram in $P$. The spatial optimality of LCA means that the target tiling by the Voronoi diagram in

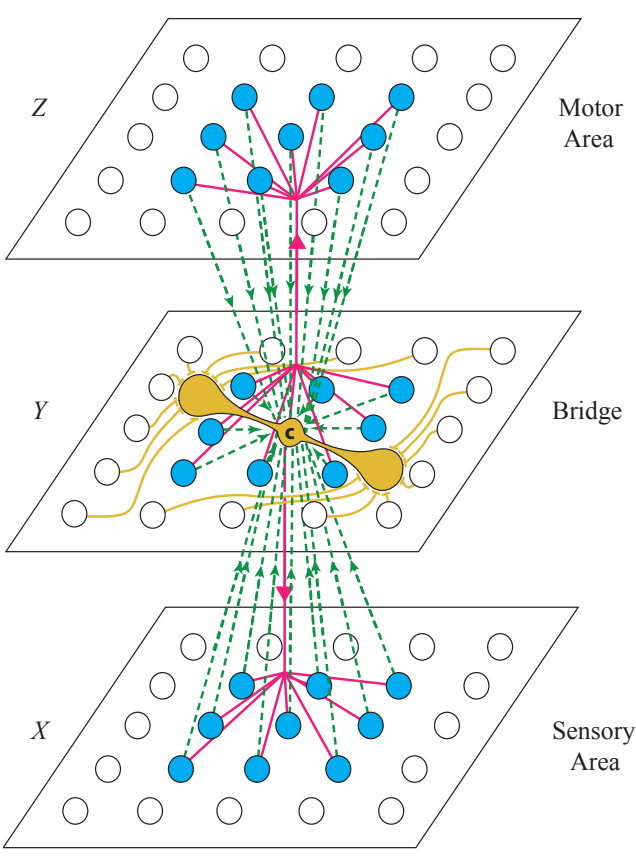

Fig. 2. An illustration of LCA network model.

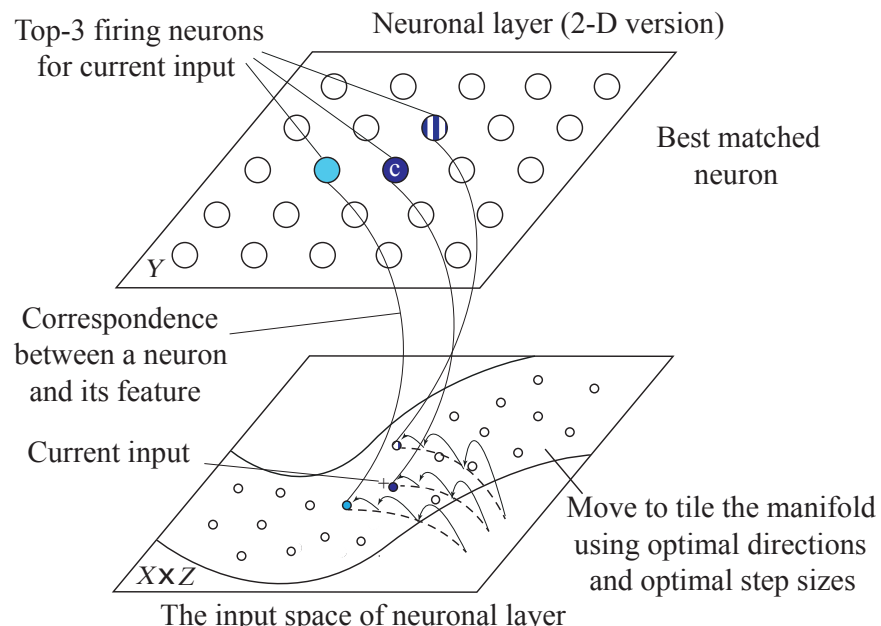

Fig. 3. The meaning of dual optimality in LCA network.

the manifold is optimal to minimize the representation error for $P=X \times Z$, whereas its temporal optimality means that the neuronal weight of firing neurons must move toward their unknown best target the quickest throughout the development process. Not only the direction but also every step size of each neuronal update is nearly optimal, as the statistical efficiency theory for neuronal weight update ensures minimum error in each age-dependent update.

\section{Where-What Network}

The "Where-What" Network (WWN), a DN embodiment, shown in Figure 4(b) shows the three areas of a simple WWN - retina $X$, simple brain $Y$, and motor $Z$. The $Z$ area has two concept areas type or "what" motor TM and location or 


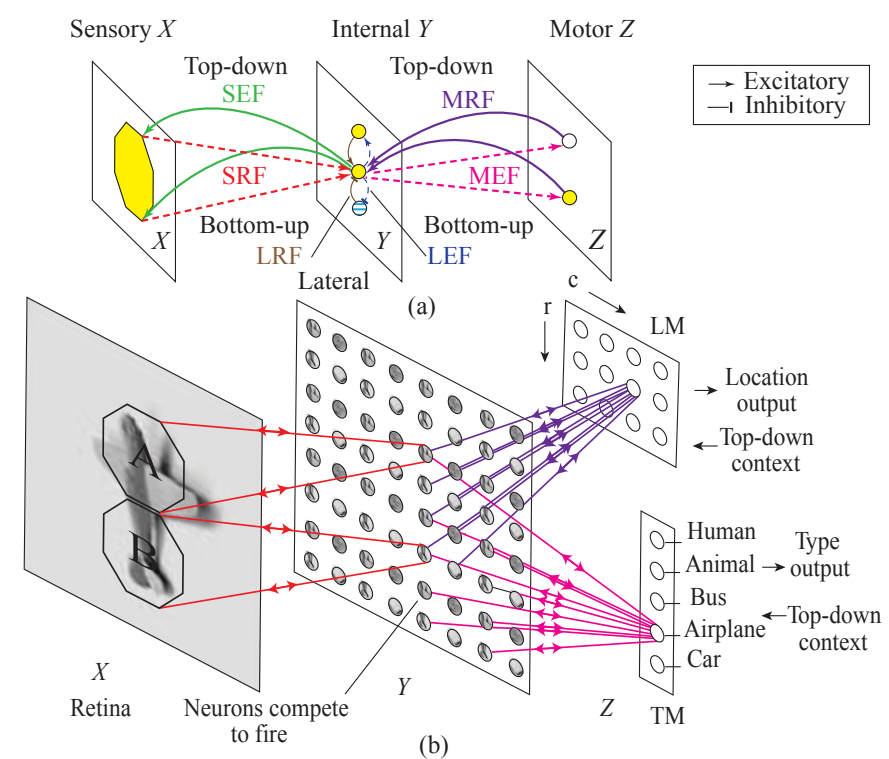

Fig. 4. Where-What networks. (a) Each neuron has 6 fields. (b) Multiple firing features contribute to location and type.

"where" motor LM. The connecting wires indicate that the presynaptic and post-synaptic neurons have co-fired; a two-way arrow indicates two one-way connections whose two synapses are generally not same. The weight is the frequency of presynaptic co-firing when the post-synaptic neuron fires. Within each cortical area, each neuron connects with highly correlated neurons using excitatory connections but with highly anticorrelated neurons using inhibitory connections. Every $Y$ neuron is location-specific and type-specific, corresponding to an object type (marked by its color pattern) and to a location block $(2 \times 2$ each). Each LM neuron is location-specific and type-invariant, and each TM neuron is type-specific and location-invariant. Each $Z$ neuron pulls all applicable cases from $Y$ area neurons as well as boosts all applicable cases in $Y$ as top-down context. A WWN does not treat features in $Y$ as a "bag-of-features" to reduce the number of training samples, because of the inner-product-based neuronal response for $Z$. The location of each element in a vector $\mathbf{x}$ affect the outcome of the inner product.

In case of shortage of $Y$ neurons, each $Y$ neuron deals with the misalignment between an object and its receptive field, simulating a more realistic resource situation. This shows that, a WWN enables un-modeled concepts, in this case, location and type, to be learned interactively and incrementally as actions, serve as attended spatiotemporal equivalent topdown contexts to direct perception, recognition and behavior emergence [10].

\section{E. Properties of $D N$}

Here, we discuss two important properties of DN distance-sensitive property and top-down representational property relevant to our work.

First, the expression for neuronal learning can be rewritten as $\mathbf{v}_{j} \leftarrow \mathbf{v}_{j}+w\left(n_{j}\right)\left(y \mathbf{p}-\mathbf{v}_{j}\right)$. Thus, the amount of vector

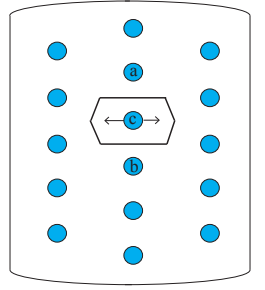

(a)

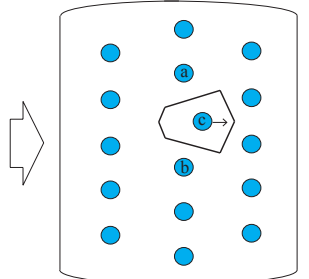

(b)

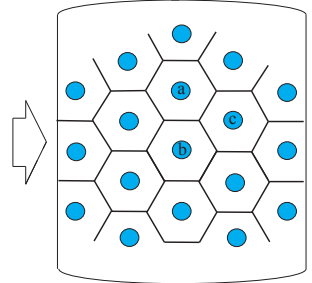

(c)
Fig. 5. The square-like tiling property of the self-organization in a cortical area

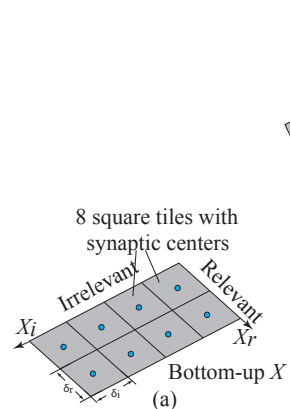

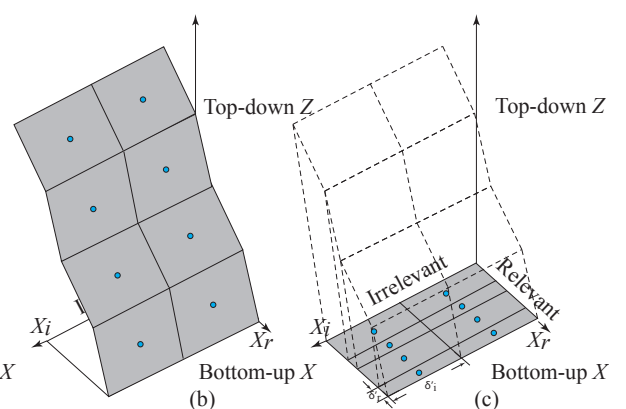

(c)
Fig. 6. The top-down representational effect: the $X_{r}$ subspace is sampled denser than $X_{i}$.

change $w\left(n_{j}\right)\left(y \mathbf{p}-\mathbf{v}_{j}\right)$ is proportional to the vector difference $y \mathbf{p}-\mathbf{v}_{j}=\mathbf{p}-\mathbf{v}_{j}$ when $y=1$, called the distance-sensitive property. With this property, [10] has established the squarelike tiling property:

Suppose that the learning rule in a selforganization scheme has the distance-sensitive property. Then the neurons in the area move toward a uniformly distribution (tiling) in the space of areal input $\mathbf{p}$ if its probability density is uniform.

The square-like tiling property of $\mathrm{DN}$ is illustrated in Figure 5. In a uniform input space, neurons in an layer self-organize until their Voronoi regions are nearly isotropic (square-like to nearly hexagons in 2-D). The Voronoi region of neuron $c$ is very anisotropic - elongated horizontally) resulting horizontal pulling is statistically stronger as shown in Figure 5(a). A horizontal perturbation leads to continued expected pulling in the same direction as shown in Figure $5(\mathrm{~b})$. Through many updates, the Voronoi regions are nearly isotropic, as illustrated in Figure 5(c).

Second, as shown in Figure 6, learning using top-down inputs sensitizes neurons to action-relevant bottom-up input (e.g., foreground pixels) and desensitize to irrelevant components (e.g., leaked-in background pixels), when top-down input is unavailable during free-viewing, called the top-down representational effect, established in [10]:

Given a fixed number of neurons in a selforganization scheme that satisfies the distance sensitivity property, adding top-down input from motor $Z$ in addition to bottom-up input $X$ enables the quantization errors for action-relevant subspace $X_{r}$ to be smaller than the action-irrelevant subspace $X_{i}$, 
where $X=X_{r} \times X_{i}$.

The top-down inputs sensitize the response for relevant bottom components although which are relevant is unknown. Without top-down input, square Voronoi tiles in the bottomup space give the same quantization width for irrelevant component $X_{i}$ and the relevant component $X_{r}: \delta_{i}=\delta_{r}$. All samples in each tile is quantized as the point (synaptic vector) at the center as illustrated in Figure 5(a). With top-down inputs during learning, square tiles cover the observed input manifold, indicating the local relationships between $X_{r}$ and $Z$ as shown in Figure 5(b). When top-down $Z$ is not available during freeviewing, each tile is narrower along direction $X_{r}$ than along $X_{i}: \delta_{r}^{\prime}<\delta_{i}^{\prime}$, meaning that the average quantization error for relevant $X_{r}$ is smaller than that for irrelevant $X_{i}$ as shown in Figure 5(c).

The above theorem gives two consequences (due to $\delta_{i}^{\prime}>$ $\left.\delta_{r}^{\prime}\right)$ : First, action-relevant bottom-up inputs are salient (e.g., toys and other Gestalt effects). Thus, we need to reconsider the conventional thinking that bottom-up saliency is and probably totally innate. Second, relatively higher variation through a synapse gives information for cellular synaptic pruning in all neurons, to delete their links to irrelevant components. This was used in synapse maintenance [11], but this capability has been turned off because the public image datasets, that we use here, do not present variation of background pixels like a dynamic physical world would.

\section{EXPERIMENTAL RESULTS}

In this section, we conduct two experiments, the DN algorithm is applied to: (a) A global template, in which the patterns to be recognized have been shifted, rotated, and scaled so that the entire input image contains mainly the pattern of interest (explained briefly). (b) A set of local templates, in which each input image contains a cluttered background, which means that the detection and pre-normalization in (a) of object of interest has not been done. The experimental results are compared to other widely-used major algorithms in the pattern recognition community.

\section{A. Global Template Based Methods}

In the first experiment, a set of well-framed "feature images" from two datasets - Weizmann and FERET are trained on LDA, SVM and LCA-net algorithms. In Weizmann dataset, image size is $88 \times 64$, from 28 humans, each having 30 images with all possible combinations of 2 expressions, 3 lighting conditions, 5 orientations, of which 812 images are used for training and 28 images are for testing. The FERET face dataset [12] is a set of 1762 images, of size $88 \times 64$, from 1010 humans, of which 1624 images are used for training and 138 images are used for testing. The LDA classifier gains $0.6 \%$ error on the Weizmann dataset after 30 cross-validation tests (a different image from each class is used for testing in each test) and $17.4 \%$ error on FERET data. SVM algorithm gives $15.3 \%$ error on FERET data using RBF kernel $(\mathrm{C}=2$; $\gamma=0.0313$ ) and $9.7 \%$ error on Weizmann data using linear kernel. LCA algorithm performs better than LDA and SVM

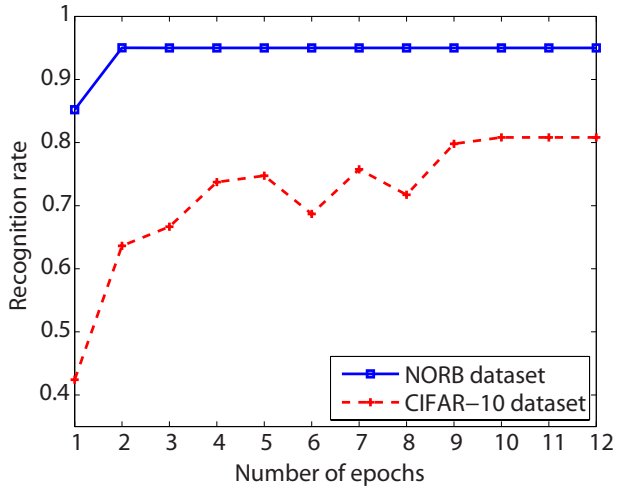

Fig. 7. The variation number of epochs in training phase to gain maximum attainable recognition rate on NORB and CIFAR-10 datasets

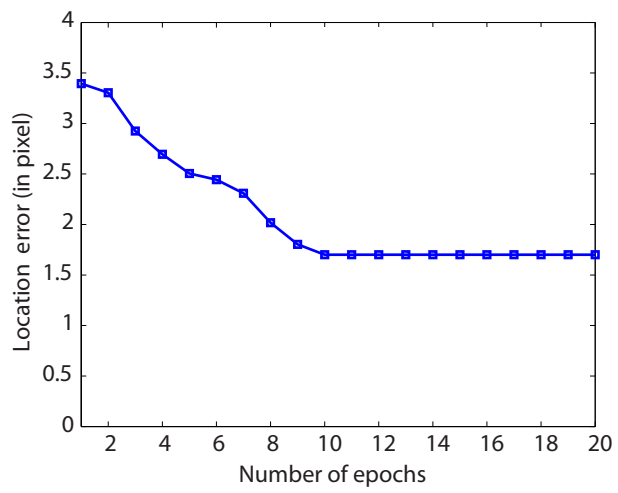

Fig. 9. The number of epochs in training phase versus the location error (in pixels) in CIFAR-10 dataset.

- using 625 neurons, it gains no error on Weizmann dataset and $8.7 \%$ error on FERET dataset.

\section{B. Local Template Based Methods}

In the second experiment, $\mathrm{DN}$ is applied to a set of local templates is derived from two object recognition datasets, NORB and CIFAR-10 using the idea of local patch extraction from foreground in [11] and the recognition rate is compared to some major local-feature learning algorithms [5], [6], [8], [9].

TABLE II

RECOGNITION RATE GAINED FROM VARIATION OF TOP-K FIRING NEURONS AND PATCH SIZE ON NORB DATASET

\begin{tabular}{lccccc}
\hline Patch size $\backslash \mathrm{k}$ & 1 & 2 & 3 & 4 & 5 \\
\hline \hline $16 \times 16$ & $48.0 \%$ & $59.8 \%$ & $85.1 \%$ & $68.0 \%$ & $54.1 \%$ \\
$17 \times 17$ & $53.1 \%$ & $60.1 \%$ & $93.8 \%$ & $72.9 \%$ & $63.5 \%$ \\
$18 \times 18$ & $53.1 \%$ & $63.5 \%$ & $93.8 \%$ & $76.0 \%$ & $63.5 \%$ \\
$19 \times 19$ & $53.1 \%$ & $63.5 \%$ & $94.0 \%$ & $81.6 \%$ & $71.9 \%$ \\
$20 \times 20$ & $63.2 \%$ & $72.6 \%$ & $94.2 \%$ & $83.2 \%$ & $71.9 \%$ \\
$21 \times 21$ & $63.2 \%$ & $72.9 \%$ & $94.2 \%$ & $83.7 \%$ & $74.5 \%$ \\
$22 \times 22$ & $69.9 \%$ & $74.5 \%$ & $95.0 \%$ & $85.2 \%$ & $74.5 \%$ \\
$23 \times 23$ & $67.1 \%$ & $71.3 \%$ & $94.2 \%$ & $81.6 \%$ & $74.2 \%$ \\
$24 \times 24$ & $61.3 \%$ & $69.8 \%$ & $94.2 \%$ & $81.6 \%$ & $66.3 \%$ \\
$25 \times 25$ & $56.2 \%$ & $68.8 \%$ & $94.0 \%$ & $79.2 \%$ & $45.0 \%$ \\
$26 \times 26$ & $43.7 \%$ & $65.2 \%$ & $93.5 \%$ & $77.5 \%$ & $32.1 \%$ \\
$27 \times 27$ & $26.4 \%$ & $65.2 \%$ & $81.2 \%$ & $51.3 \%$ & $24.7 \%$ \\
$28 \times 28$ & - & $49.1 \%$ & $74.9 \%$ & $34.7 \%$ & - \\
$29 \times 29$ & - & $49.1 \%$ & $74.9 \%$ & $27.8 \%$ & - \\
\hline
\end{tabular}




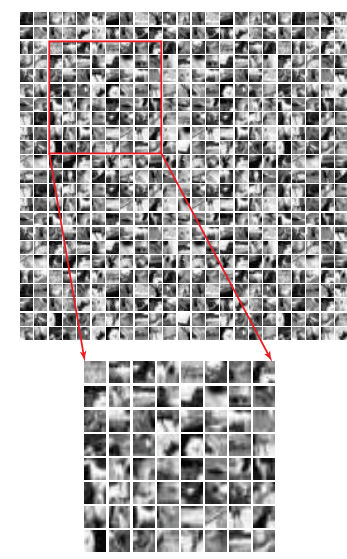

(a) Bottom- up $Y$

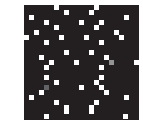

"Ship" neuron

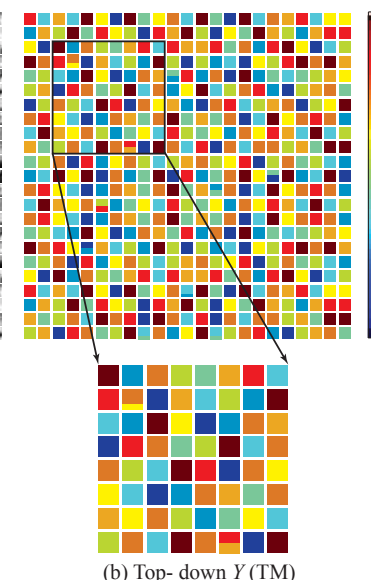

(b) Top- down $Y$ (TM)

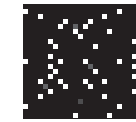

"Automobile" neuron

(d) Bottom- up $Z$

R
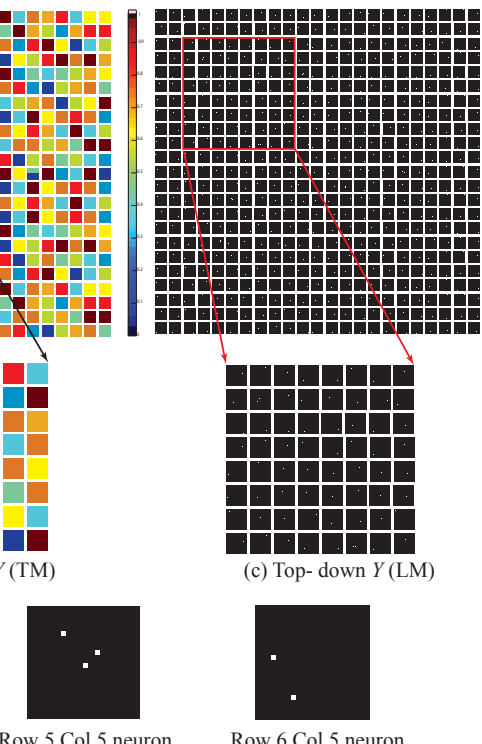

Fig. 8. Visualization of weights in one depth (of 30 depths) in $Y$ area of CIFAR-10 (a) bottom-up weights (23 $\times 23 Y$ neurons in which each cell has dimension $10 \times 10)($ b) top-down weights $(\mathrm{TM})(23 \times 23 \mathrm{TM}$ neurons in which each cell has dimension $10 \times 10)(\mathrm{c})$ top-down weights $(\mathrm{LM})(23 \times 23 \mathrm{LM}$ neurons in which each cell has dimension $23 \times 23$ ) (d) Visualization of the bottom-up weights for $Z$ area - two type neurons in one depth (of 30 depths) in TM $(1 \times 10$ TM neurons in which each cell has dimension $23 \times 23)$ and two location neurons in LM $(23 \times 23$ LM neurons in which each cell has dimension $23 \times 23)$.

TABLE III

RECOGNITION RATE GAINED FROM VARIATION OF TOP-K FIRING NEURONS AND PATCH SIZE ON CIFAR-10 DATASET

\begin{tabular}{lccccc}
\hline Patch size $\backslash \mathrm{k}$ & 1 & 2 & 3 & 4 & 5 \\
\hline \hline $5 \times 5$ & - & - & $25.2 \%$ & - & - \\
$6 \times 6$ & - & $22.2 \%$ & $39.4 \%$ & - & - \\
$7 \times 7$ & - & $30.3 \%$ & $51.5 \%$ & $23.2 \%$ & - \\
$8 \times 8$ & $25.2 \%$ & $30.3 \%$ & $64.6 \%$ & $38.3 \%$ & - \\
$9 \times 9$ & $36.3 \%$ & $44.4 \%$ & $72.7 \%$ & $52.5 \%$ & $29.3 \%$ \\
$10 \times 10$ & $36.3 \%$ & $44.4 \%$ & $80.8 \%$ & $63.6 \%$ & $43.4 \%$ \\
$11 \times 11$ & $35.3 \%$ & $42.4 \%$ & $79.8 \%$ & $61.6 \%$ & $42.4 \%$ \\
$12 \times 12$ & $36.3 \%$ & $44.4 \%$ & $76.7 \%$ & $59.6 \%$ & $43.4 \%$ \\
$13 \times 13$ & $36.3 \%$ & $44.4 \%$ & $71.7 \%$ & $56.5 \%$ & $43.4 \%$ \\
$14 \times 14$ & $32.3 \%$ & $43.4 \%$ & $68.6 \%$ & $53.5 \%$ & $40.4 \%$ \\
$15 \times 15$ & $32.3 \%$ & $43.4 \%$ & $67.6 \%$ & $51.5 \%$ & $40.4 \%$ \\
$16 \times 16$ & $29.3 \%$ & $40.4 \%$ & $64.6 \%$ & $49.5 \%$ & $40.4 \%$ \\
\hline
\end{tabular}

The NORB dataset with elimination of complex background [13] is a set of images of 50 toys, of size $96 \times 96$, belonging to 5 classes namely, four-legged animals, human figures, airplanes, trucks and cars, imaged by two cameras under 6 lighting conditions, 9 elevations and 18 azimuths, of which 24300 images were used for training and testing each. The CIFAR-10 dataset [14] is a set of 60000 color images, of size $32 \times 32$, belonging to 10 classes, with 6000 images per class, of which 50000 images are used for training 10000 images are used for testing. Each image in the training set of NORB images is rotated at every angle of $20^{\circ}$, so that 18 rotated instances of each image in training dataset are obtained, which are added to the original training set so that a test image is correctly classified regardless of the elevation angle. In the CIFAR-10 dataset, each patch extracted from the training image is trained at every location within the image, for instance, a patch of size 10 is trained at $(32-10+1) \times(32-10+1)$ i.e.
$23 \times 23$ different locations within the image and each of the shifted image instances are added to the training set, so that a test image is correctly classified regardless of its position within the image.

TABLE IV

LOCAL TEMPLATE BASED METHOD COMPARISON ON NORB DATASET

\begin{tabular}{lc}
\hline Algorithm & Recognition rate \\
\hline \hline Conv. Neural Network & $93.4 \%$ \\
Deep Boltzmann Machine & $92.8 \%$ \\
Deep Belief Network & $95.0 \%$ \\
Deep Neural Network & $97.1 \%$ \\
\hline Sparse Auto-encoder & $96.9 \%$ \\
Sparse RBM & $96.2 \%$ \\
K-means (Hard) & $96.9 \%$ \\
K-means (Triangle) & $97.0 \%$ \\
K-means (Triangle, 4000 features) & $97.2 \%$ \\
\hline WWN & $95.0 \%$ \\
per frame training time & $1.3 \mathrm{~s}$ \\
per image testing time & $0.8 \mathrm{~s}$ \\
\hline
\end{tabular}

TABLE V

LOCAL TEMPLATE BASED METHOD COMPARISON ON CIFAR-10 DATASET

\begin{tabular}{lcc}
\hline Algorithm & Rec rate & Loc Err (pixels) \\
\hline \hline Raw pixels & $37.3 \%$ & - \\
3-Way Factored RBM (3 layers) & $65.3 \%$ & - \\
Mean-covariance RBM (3 layers) & $71.0 \%$ & - \\
Improved Local Coord. Coding & $74.5 \%$ & - \\
Conv. Deep Belief Net (2 layers) & $78.9 \%$ & - \\
\hline Sparse Auto-encoder & $73.4 \%$ & - \\
Sparse RBM & $72.4 \%$ & - \\
K-means (Hard) & $68.6 \%$ & - \\
K-means (Triangle) & $77.9 \%$ & - \\
K-means (Triangle, 4000 features) & $79.6 \%$ & - \\
\hline WWN & $80.8 \%$ & 1.7 \\
per frame training time & $1.5 \mathrm{~s}$ & \\
per image testing time & $1.2 \mathrm{~s}$ & \\
\hline
\end{tabular}


The local feature templates derived from NORB and CIFAR-10 images are trained on the WWN algorithm [11] A recognition rate of $95.0 \%$ was obtained from NORB dataset for a patch of size $22 \times 22$, the thickness of the network being 10, when top-3 neurons fire as shown in Table II; whereas a recognition rate of $80.8 \%$ was obtained from CIFAR-10 images for a patch of size $10 \times 10$, the thickness of the network being 30, when top-3 neurons fire as shown in Table III. The number of training epochs are varied from 0 to 15 and the recognition rate at each epoch is plotted as shown in Figure 7. Each epoch performs 3 iterations for reinforcement learning of LM and TM input by WWN. The internal representation of $Y$ area and $Z$ area after the training phase of the WWN algorithm are visualized in Figure 8. Each $Y$ neuron (in all depths) detects a type as in Figure 8(b) in a specific location as in Figure 8 (c). Due to limited neuronal resources in $Y$ area, some neurons deal with multiple object types at multiple pixel locations. The bottom-up weights (TM and LM) of two of $Z$ neurons as in Figure 8 (d) are normalized to the range 0 to 255 such that the pixel value indicates the strength of the connection between the corresponding $Y$ neuron (the same (row,col) location as the pixel) and the $Z$ neuron. The Figure 9 shows the location error (in pixels) over 20 epochs for CIFAR10; the location error remains constant after 10 epochs. The recognition rate, thus obtained, is compared to the recognition rate obtained by some major local-feature learning algorithms as shown in Table IV and Table $\mathrm{V}$ to show that a WWN performs comparable to them.

In NORB dataset, the object of interest is already centered, thus, "where" information in DN gets no room for improvement. However, in the CIFAR-10 dataset, the object of interest appears at different locations within a scene. Thus, the "where" information from LM ensures that the object is present in the scene as a configuration (not necessarily rigid) that is consistent with the training experience, not as scattered broken parts, for instance, the head and the tail of an airplane is present with an observed configuration and not as separate disassembled parts, which might be a reason for the recognition rate to be slightly better than other methods.

\section{CONCLUSIONS}

In this work, the comparison of DN algorithm major wellknown pattern recognition algorithms showed that the performance of the DN method is comparable to those major existing methods. In the local template based problems, DN allows different firing feature neurons to vote for a single neuron in TM and LM, which has reached pixel-level location accuracy for CIFAR-10 dataset. The work here indicates that the pay-off of finding the better spatial features seems to have diminished for static image-based methods. The DN method used here, for space-only problem was meant for a tight integration of space and time information for visual events in natural cluttered backgrounds, where objects dynamically sweep across dynamic backgrounds and other foregrounds. In the work of [15], [16], it has been shown that using temporal information for spatial recognition in DN has considerably improved its recognition rate without imposing rigid constraints on object appearance through time, which is typical with model-based object tracking.

A possible future direction of research is the detection of object contours from richly textured dynamic background using a technique called synapse maintenance [11]. The function of synapse maintenance in DN was not used for experiments here because the image datasets available in the public repositories consist of snapshots of static scenes which are very different from what human-eye sees from the dynamic physical world, where the contours of unknown objects manifest themselves when an object moves relative with its cluttered dynamic backgrounds.

\section{ACKNOWLEDGMENT}

The authors would like to thank Matthew Luciw and Yuekai Wang for providing some of their programs.

\section{REFERENCES}

[1] R. Fisher, "The use of multiple measurements in taxonomic problems," Annals of Eugenics, vol. 7, pp. 179-188, 1936.

[2] D. L. Swets and J. Weng, "Using discriminant eigenfeatures for image retrieval," IEEE Trans. Pattern Analysis and Machine Intelligence, vol. 18 , no. 8, pp. 831-836, 1996.

[3] C. Cortes and V. Vapnik, "Support-vector networks."

[4] T. Poggio and G. Federico, "Networks for approximation and learning," Proceedings of The IEEE, vol. 78, no. 9, pp. 1481-1497, 1990.

[5] I. Goodfellow, Q. Le, A. Saxe, H. Lee, and A. Ng, "Measuring invariances in deep networks," NIPS, 2009.

[6] H. Lee, R. Grosse, R. Ranganath, and A. Y. Ng, "Convolutional deep belief networks for scalable unsupervised learning of hierarchical representations," in Proc. 26th Int'l Conf. on Machine Learning, Montreal, Canada, June 14-18 2009, pp. 609-616.

[7] B. A. Olshaushen and D. J. Field, "Emergence of simple-cell receptive field properties by learning a sparse code for natural images," Nature, vol. 381, pp. 607-609, June 131996.

[8] J.Yang, K. Yu, Y. Gong, and T. Huang, "Linear spatial pyramid matching using sparse coding for image classification," Computer Vision and Pattern Recognition, 2009.

[9] A. Coates, H. Lee, and A. Ng, "An analysis of single-layer networks in unsupervised feature learning," in Proceedings of the 14th International Conference on Artificial Intelligence and Statistics (AISTATS), vol. 15 of JMLR: W\&CP 15, Fort Lauderdale, FL, USA, 2011.

[10] J. Weng and M. Luciw, "Brain-like emergent spatial processing," IEEE Trans. Autonomous Mental Development, vol. 4, no. 2, pp. 161-185, 2012.

[11] Y. Wang, X. Wu, and J. Weng, "Synapse maintenance in the where-what network," in Proc. Int'l Joint Conference on Neural Networks, San Jose, CA, July 31 - August 5 2011, pp. 2823-2829.

[12] P. J. Phillips, H. Moon, P. Rauss, and S. A. Rizvi, "The FERET evaluation methodology for face-recognition algorithms," in Proc. IEEE Conf. Computer Vision and Pattern Recognition, Puerto Rico, June 1997, pp. 137-143.

[13] Y. LeCun, F. Huang, and L. Bottou, "Learning methods for generic object recognition with invariance to pose and lighting," IEEE Computer Society Conference on Computer Vision and Pattern Recognition (CVPR), 2004.

[14] A. Krizhevsky, "Learning multiple layers of features from tiny images," Department of Computer Science, University of Toronto: Master's thesis, 2009.

[15] M. Luciw and J. Weng, "Where What Network 3: Developmental topdown attention with multiple meaningful foregrounds," in Proc. IEEE Int'l Joint Conference on Neural Networks, Barcelona, Spain, July 18-23 2010, pp. 4233-4240.

[16] J. Weng, "Why have we passed "neural networks do not abstract well"?" Natural Intelligence: the INNS Magazine, vol. 1, no. 1, pp. 13-22, 2011. 